\title{
The Influence of Empathy in Human-Robot Relations
}

\author{
Iolanda Leite*, André Pereira, Samuel Mascarenhas, Carlos Martinho, Rui Prada, Ana Paiva \\ INESC-ID and Instituto Superior Técnico \\ Av. Prof. Cavaco Silva, Taguspark 2744-016, Porto Salvo, Portugal
}

\begin{abstract}
The idea of robotic companions capable of establishing meaningful relationships with humans remains far from being accomplished. To achieve this, robots must interact with people in natural ways, employing social mechanisms that people use while interacting with each other. One such mechanism is empathy, often seen as the basis of social cooperation and prosocial behaviour. We argue that artificial companions capable of behaving in an empathic manner, which involves the capacity to recognise another's affect and respond appropriately, are more successful at establishing and maintaining a positive relationship with users. This paper presents a study where an autonomous robot with empathic capabilities acts as a social companion to two players in a chess game. The robot reacts to the moves played on the chessboard by displaying several facial expressions and verbal utterances, showing empathic behaviours towards one player and behaving neutrally towards the other. Quantitative and qualitative results of 31 participants indicate that users towards whom the robot behaved empathically perceived the robot as friendlier, which supports our hypothesis that empathy plays a key role in human-robot interaction.
\end{abstract}

Keywords: Artificial companions, social robots, empathy, affective interactions, friendship.

\section{Introduction}

Recent research in social robots is transforming a once science fiction topic into a scientific domain. However, there is still a long way to go for robots to become as socially intelligent and unique as those described in science fiction stories. On the one hand, the physical appearance of robots has undergone remarkable recent improvements (Ishiguro, 2008). On the other hand, we remain far from a time where robots can emulate the complex social dynamics found in humans in a highly believable manner.

Existing artificial companions have limited abilities to support meaningful social interactions with users in real social settings. To improve the interactions between humans and artificial companions, the LIREC Project (Living with Robots and Interactive Companions) ${ }^{1}$ aims to create a new generation of interactive, emotionally intelligent companions capable of engaging users in long-term interactions. The application domains where these companions can assist users are innumerable, from cognitive assistants for the elderly to educational companions for children (Breazeal, 2009).

As social beings, relationships are important to humans. Empathy facilitates the creation and development of social relationships (Anderson and Keltner, 2002), as it increases similarity, fondness and affiliation (De Vignemont and Singer, 2006). Empathy can be broadly defined as "an affective response more appropriate to someone else's situation than to one's own" (Hoffman, 2001, p. 4). Previous work showed that empathic agents are perceived as more caring, likeable, and trustworthy than agents without empathic capabilities (Brave et al., 2005) and that empathic agents can foster empathic feelings in users (Paiva et al., 2004). In Human-Robot Interaction, interest has recently increased in studying different forms of empathy on users' attitudes towards robots (Tapus and Mataric, 2007; Cramer et al., 2010; Riek et al., 2010).

\footnotetext{
${ }^{*}$ Corresponding author

E-mail address: iolanda.leite@ist.utl.pt

${ }^{1}$ http://lirec.eu/ 
Our goal is to study which aspects of social interaction are relevant for robots that interact with users over extended periods of time. The motivation for studying empathy comes not only from the extensive literature highlighting the role of empathy in social relations, but also from a previous long-term study where we investigated users' perceived social presence towards a social robot over a five-week period (Leite et al., 2009). In this study, the social presence dimension that decreased the most over time was perceived affective interdependence, defined as the extent to which users believe that the affective state of the robot is influenced by their own affective state. Continuing this line of research, this paper aims to investigate the following hypothesis: people towards whom a social robot behaves empathically provide better ratings of perceived friendship than people towards whom the robot behaves neutrally. We decided to measure perceived friendship because, as argued earlier, empathy fosters the creation and development of social bonds between people. Such bonds can originate, among other types of social relations, friendship. To test the presented hypothesis, we developed a scenario where a social robot with empathic capabilities observes two humans playing a chess match against each other, reacting emotionally and verbally to the moves played by both players. However, the robot empathises only with one player, behaving neutrally towards the other. In this paper, we report the findings of a study that we have conducted where 40 subjects interacted with this robot.

This paper is structured as follows. We begin by presenting some theoretical background on empathy and a brief overview on the existing work on empathic robots. Then, we explain how we infer the user's affective state and the empathic behaviours that the robot performs in response to those states. We then describe an experiment based on our application scenario and present our results. Finally, we discuss the obtained results and some future work directions.

\section{Theoretical Background}

Although scholars seem to agree on the importance of empathy in fostering prosocial behaviour (Feshbach, 1978; Batson and Shaw, 1991) and developing social relationships (Stephan and Finlay, 1999), the concept itself has a history of definitional disagreement and ambiguity (Einsenberg, 2000). According to Strayer (1987) and Davis (1996), the innumerable attempts at defining empathy fall under two categories: an affective or cognitive approach. In the latter, empathy is usually defined as the ability to cognitively understand anothers' feelings and thoughts through perspective-taking, i.e. the ability of "putting oneself in the other's place and imagining how he or she feels" (Hoffman, 2001, p. 52). Defined in this manner, empathy does not necessarily require the person to feel an emotional state as a result. In contrast, empathy is defined in the affective approach as "an affective response more appropriate to another's situation than one's own" (Hoffman, 2001, p. 4) or "an observer's reacting emotionally because he perceives that another is experiencing or is about to experience an emotion"(Stotland, 1969, p. 272). In this perspective, empathy may occur by means that require no cognitive effort, such as bodily mimicry of another person's behaviour including, but not limited to, facial expressions and body postures.

The separation between cognitive and affective empathy seems to be useful from a theoretical perspective. However, the two groups overlap to some extent. This is clearly evident in some definitions of empathy, including that proposed by Hoffman (2001), in which empathy is defined as having an affective nature that may result from cognitive processes such as perspective-taking. More precisely, he defines empathy as an affective response that results from one (or more) of the following empathic arousal mechanisms: motor mimicry, classical conditioning, direct association of cues from others, mediated association (association of cues from others through semantic processing) and perspective-taking. The first three are considered preverbal, automatic and involuntary, and the last two are higherorder cognitive modes. From these five empathic arousal mechanisms, there is empirical evidence suggesting that perspective-taking is more "empathy-arousing" than focusing directly on verbal and non-verbal cues (Stotland, 1969). Perspective-taking has thus been considered an important component of the empathic process (Feshbach, 1978). Motivated by these findings, the robot's empathy in our work results from perspective-taking but still contains an affective response and not merely an understanding of what the companion is feeling. The robot appraises the game context considering its companion point of view and generates an affective state congruent with the companion's situation.

Although empathy can be seen as an intrinsic characteristic of human behaviour, external factors can sometimes limit the prosocial behaviours triggered by empathic feelings. Hoffman (2001) argues that such limitations are due to two main reasons: empathic over-arousal and relationship between the observer and empathy target. Empathic over-arousal occurs when the signs of distress are so intense that the observer's empathic distress is transformed into a feeling of personal distress. The relationship between the observer and empathy target also strongly affects the type of prosocial action taken by the observer. For example, people tend to be more empathic towards friends and family 
members than towards strangers (Krebs, 1970). Other researchers suggest that empathic responses can be modulated by other factors, including the observer's personal characteristics (e.g., past experience, mood, personality, gender, age) or situational context (De Vignemont and Singer, 2006). In our work, the empathic responses of the robot are modulated by the relationship between the robot and the players.

\section{Related Work}

Empathy is a research topic that has received a lot of attention in the field of virtual agents (Paiva et al., 2004; Prendinger and Ishizuka, 2005; Bickmore and Picard, 2005; Brave et al., 2005; McQuiggan and Lester, 2007). However, the first studies in social robotics addressing empathy have only recently started to appear. One possible reason for this delay is that it is often easier to infer the user's affective state in interaction scenarios with virtual agents than it is with human-robot scenarios. When interacting with virtual agents, the user usually sits in front of a computer screen, and there is often the possibility of selecting predefined dialogues to inform the agent of the user's emotional state, as in (Bickmore and Picard, 2005). Interaction with robots tends to be more open-ended, and perceiving the user's affective state is thus a more challenging task. Nevertheless, there have been some significant improvements regarding automatic affect recognition using different modalities, including vision, speech or physiological signals (Zeng et al., 2009).

In social robotics, most research addressing empathy has focused on mimicking the user's affective state, a particular aspect of empathy also designated as emotional contagion. For instance, Hegel et al. (2006) presented a study with an anthropomorphic robot that recognises the user's emotional state through speech intonation and mirrors the inferred state using a corresponding facial expression. The results suggested that users who interacted with this version of the robot found the robot's responses more adequate both in its appropriateness to the social situation and timing than subjects who interacted with the robot without affective expressions. In another study (Riek et al., 2010), a robot with the form of a chimpanzee head mimicked the user's mouth and head movements. When interacting with this robot, most subjects considered the interaction more satisfactory than participants who interacted with a version of the robot without mimicking capabilities.

Recently, a study was conducted to assess how empathy affects people's attitudes towards robots (Cramer et al., 2010). Using a between-subjects design, two groups of participants saw a four-minute video with an actor playing a cooperative game with an iCat robot. In one condition, the robot accurately expressed empathic behaviour toward the actor, whereas in the other condition, the robot inaccurately expressed empathic behaviour toward the actor (i.e., incongruent to the situation). In this study, there was a significant negative effect on user's trust in the inaccurate empathic behaviour condition. Conversely, participants who observed the robot displaying accurate empathic behaviours perceived their relationship with the robot as closer. This study has some similarities to the one described in this paper, but differences occur concerning the interaction modality and measures. The evaluation in this earlier study was video-based, whereas users in our study interacted directly with the robot; similarly, while this study employed measures such as the trust in and credibility of the robot, our study measured perceived friendship.

A relevant finding in empathy research is that empathy is positively linked to prosocial behaviour (Eisenberg and Fabes, 1990; Hoffman, 1982). In another study using the iCat robot, Saerbeck et al. (2010) investigated the effects of social supportive behaviours of the robot on students' learning performance and motivation. The results indicate that simple manipulations of the robot's supportiveness (while maintaining the same learning content) increased student's motivation and scores on a language test.

Our goal is to develop an empathic robot companion capable of establishing a positive social relationship with users. As such, in the attempt to achieve this goal, we took inspiration from social sciences and virtual agents research and propose a model of empathy that involves perspective-taking, where the robot tries to infer the user's emotional state by putting itself in the user's situation. This approach is similar to that proposed by Rodrigues et al. (2009), but it considers the user as the target for self-projection rather than another virtual agent. There are also some examples of perspective-taking in HRI with the goal of, for example, improving the robot's effectiveness in learning simple tasks from ambiguous demonstrations (Breazeal et al., 2006) or facilitating human-robot communication (Torrey et al., 2010). 


\section{Modelling Empathy in a Robot Companion}

To model empathy in a social robot, we developed a scenario where an iCat robot (van Breemen et al., 2005) watches and comments on a chess match between two human players. The iCat comments on every move played on the chessboard, displaying empathic behaviours towards one player, its "companion", and providing neutral comments for the other player. Many researchers (e.g., Eisenberg and Fabes 1990; Hoffman 2001) agree that empathy can be achieved by two distinct phases: (1) inferring the other's affective state and (2) responding empathically to that state.

Figure 1 presents an overview of the empathic model that we implemented in the iCat robot. When a new game event is received (i.e., when a valid move is played on the chessboard), the iCat infers the companion's affective state using perspective taking, that is, analysing the game considering the companion's point of view. The perspectivetaking analysis influences the iCat's affective state, as the robot is intended to "feel" empathy for the companion player. The robot's empathic affective state then determines its empathic response, which is modulated by the relationship with the players. The responses are thus different whether the iCat is addressing its companion or the other player. In the following subsections, we describe in more detail how the perspective-taking mechanisms and empathic responses were implemented in the iCat.

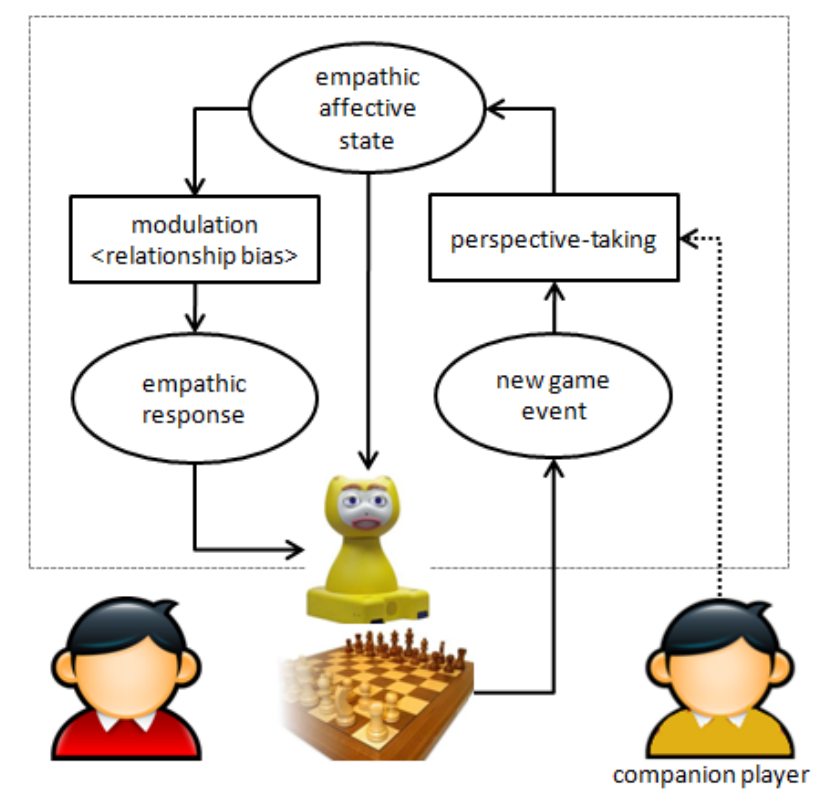

Figure 1: Overview of the empathic model. When the state of the game changes, the robot updates its affective state by inferring the companion's affective state using perspective-taking. The empathic responses of the robot vary depending on the player who made the last move on the board.

\subsection{Inferring the user's affective state: a perspective-taking approach}

Following the literature on empathy research, the capacity of assuming the perspective of another person is a valid way of inferring that person's affective state. Our robot uses a perspective-taking approach to infer the possible affective states of its companion. The iCat may have a different perspective of the game than the companion, which may lead to wrong interpretations, but the same can also happen with humans. Previous work on automatic affect recognition has also shown that the contextual information of a chess game is enough to discriminate some of the user's affective states (Castellano et al., 2009).

After every move played on the chessboard, the robot evaluates the new board position using a chess heuristic function (from the perspective of the companion player) and interprets it as if it were its own move. These values are the input of the robot's affective state, which is based on an anticipatory mechanism that generates an affective state based on the mismatch between an expectation of the next state of the game (expected value) and the real evaluation (sensed value) of the new board position (Martinho and Paiva, 2006; Leite et al., 2008). The expected value is 
computed based on the history of previous values obtained from the chess evaluation function, and the sensed value is the result of the current evaluation of the game. Using this mechanism, one of nine different affective states can be generated. For example, suppose that the companion is losing the game and plays a bad move; the iCat's consequent affective state is "expected punishment", meaning that the current state is bad, as the robot was expecting. Table 1 describes the list of possible empathic states and their meanings.

\subsection{Empathic Responses}

The affective state of the iCat influences the empathic responses the robot displays to the players. Empathic responses can range from simple facial expressions (often mimicking the other's facial expression) to more complex cognitive actions, including prosocial behaviours. However, the concrete prosocial behaviours associated with empathy are still not clearly defined. Eisenberg and Fabes (1990) attribute this to the "failure of many investigators to differentiate among various modes of prosocial behaviour". Nevertheless, empirical studies that describe typical empathic and prosocial behaviours in particular contexts are starting to appear.

To re-create empathic responses in our robot, we took inspiration from one of such studies (Cooper et al., 1999), where different characteristics of empathic teachers were identified and grouped by the following categories: bodylanguage, positioning, teaching content, teaching method, voice, attitudes, facial characteristics and verbal responses. Given the limitations of our scenario in terms of the robot's embodiment and interaction context, we only considered differences in facial characteristics and verbal responses.

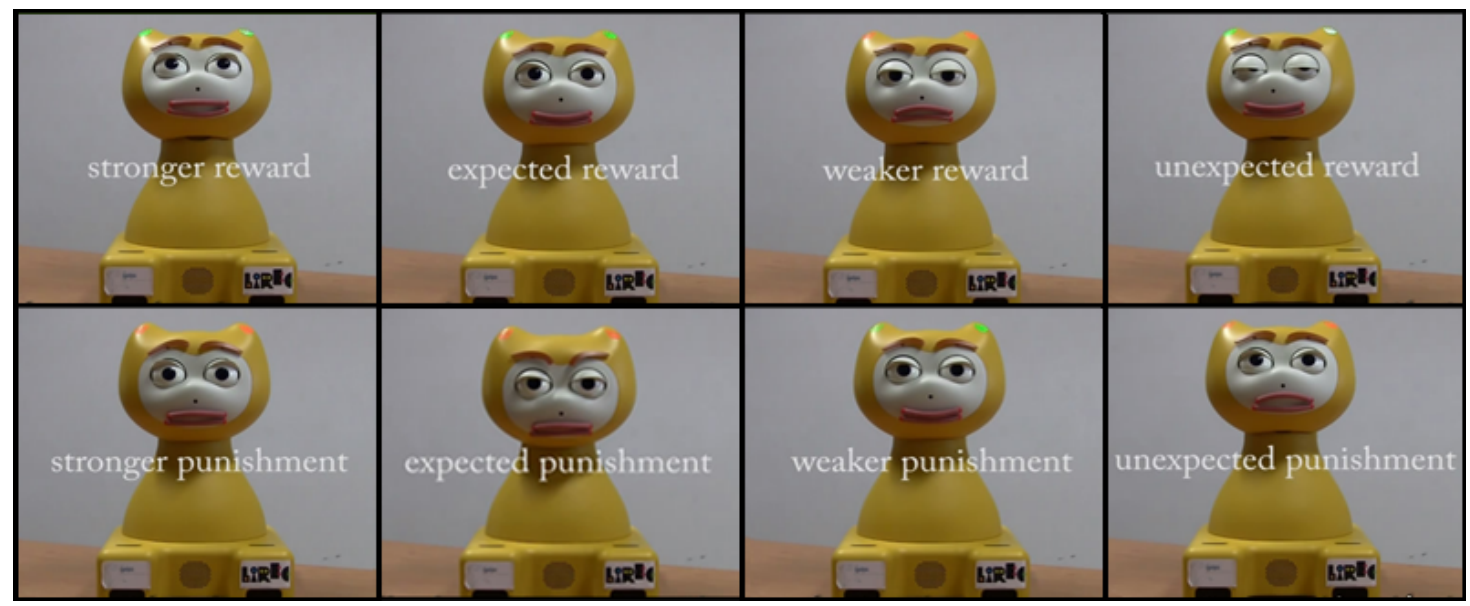

Figure 2: Snapshots of the different empathic expressions of the iCat.

\subsubsection{Facial Expressions}

Empathic teachers constantly reflect the student's emotions in their facial expressions, while non-empathic teachers are often not in tune with what they are saying. Likewise, our robot expresses its empathic state using a facial expression that tries to reflect the companion's affective state by assessing his/her situation in the game. For instance, if the companion is constantly playing bad moves and plays another bad move, the iCat's affective state would return the empathic state of "expected punishment", which means that the change in the current state of the game is bad for the companion player, and the robot was already expecting that. In this case, the iCat would express a low intensity (because it was already expected) sad expression as a result of its empathy towards the disadvantaged companion. However, as the robot analyses the game and reacts according to the companion player's perspective, if the other player makes a mistake in his/her next move, causing the companion to recover some disadvantage, the iCat's affective state would change to "weaker punishment". In this situation, the iCat would display a subtle positive facial expression congruent with the possible affective state of the companion. Figure 2 presents a complete overview of the robot's empathic facial expressions. 
Empathic teachers also tend to use more eye contact. This characteristic was also modelled in our robot: while players are pondering their next moves, the iCat displays idle behaviours, such as looking at the chessboard or the players. These behaviours were weighted such that the robot looks at its companion twice as often as the other player.

\subsubsection{Verbal Comments}

As mentioned above, empathy is positively correlated with prosocial and helping behaviours. We modelled prosocial behaviours in our robot by providing verbal feedback to the players. However, as our aim is to vary the empathic responses based on the relationship of the iCat with different players, the verbal comments depend not only on the iCat's empathic state, but also on the player who made that move. In other words, the verbal empathic responses of the robot are modulated by the relationship between the robot and players.

After reacting empathically to an event on the chessboard, the robot verbally comments on that move. As the comments depend on the iCat's empathic state and on the player who made that move, two sets of utterances were defined for each affective state: empathic utterances, to be used when the robot addresses its companion, and neutral utterances, to be used when the robot comments on the moves of the other players. While neutral utterances merely indicate the quality of the move in a very straightforward way (e.g., "bad move", "you played well this time"), empathic utterances often contain references to the companion's possible feelings in the attempt to encourage and motivate the companion (e.g., "you're doing great, carry on!"). Table 1 contains examples of possible utterances of the robot in both conditions.

As with eye contact, when speaking to its companion, the iCat uses the companion's name twice as often as when speaking to the other player. In addition to feedback after the moves, the iCat also congratulates the companion when she/he captures an opponent's piece and encourages her/him in the critical moments of the game - for example, when the chances of winning (or losing) become evident. At the end of the game, the robot's behaviour also differs depending on who won the game. If the companion wins, the robot says "Congratulations! You are a very good chess player!" (while displaying a happy facial expression); if the other player wins it simply says "Checkmate, see you on the next match" (displaying a sad facial expression).

Table 1: Description of the possible affective sensations experienced by the iCat, with examples of the verbal comments of the robot for both conditions. In the neutral utterances, the comments only vary depending on "reward" and "punishment" sensations. The utterances are sometimes combined with the name of the players either at the beginning or end of the sentence.

\begin{tabular}{|c|c|c|c|}
\hline \multirow{2}{*}{ Affective State } & \multirow{2}{*}{ Meaning } & \multicolumn{2}{|c|}{ Examples of Verbal Utterances } \\
\hline & & Empathic & Neutral \\
\hline Stronger Reward & Better than expected & $\begin{array}{l}\text { "Great move! Even better } \\
\text { than I was expecting!" }\end{array}$ & \multirow{4}{*}{$\begin{array}{l}\text { "Good move." } \\
\text { "You played well } \\
\text { this time." }\end{array}$} \\
\hline Expected Reward & As good as expected & $\begin{array}{l}\text { "Nice move, you played what } \\
\text { I would have played!" }\end{array}$ & \\
\hline Weaker Reward & Not as good as expected & $\begin{array}{l}\text { "I believe you could } \\
\text { have played better." }\end{array}$ & \\
\hline Unexpected Reward & Good, unexpected & "You played very well." & \\
\hline Unexpected Punishment & Bad, unexpected & $\begin{array}{l}\text { "Oh... I wasn't } \\
\text { expecting that move..." }\end{array}$ & \multirow{4}{*}{$\begin{array}{l}\text { "This move wasn't } \\
\text { that good." } \\
\text { "Bad move." }\end{array}$} \\
\hline Weaker Punishment & Not as bad as expected & $\begin{array}{l}\text { "Well done, you are } \\
\text { recovering your disadvantage!" }\end{array}$ & \\
\hline Expected Punishment & Bad, as expected & $\begin{array}{l}\text { "Don't worry, you didn't } \\
\text { have better options." }\end{array}$ & \\
\hline Stronger Punishment & Worse than expected & $\begin{array}{l}\text { "You're making me sad... } \\
\text { you could have played better." }\end{array}$ & \\
\hline
\end{tabular}




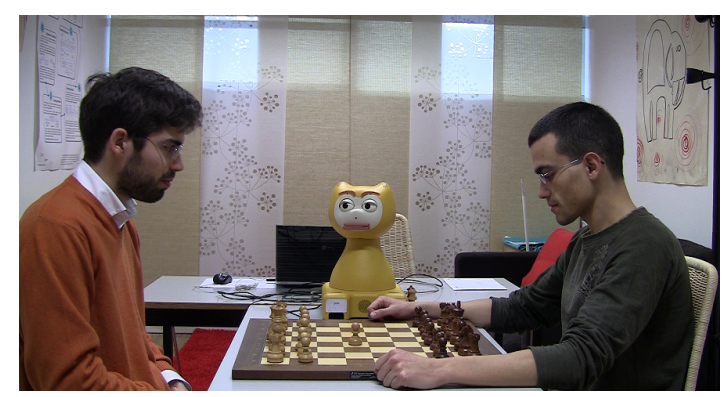

Figure 3: Users interacting with the iCat.

\section{Experiment}

In the previous section, we presented a model for a social robot that empathises with one player of a chess match by inferring his/her affective state using perspective-taking. The empathic responses of the robot vary depending on the relationship between the robot and the players. Using this scenario, we conducted an experiment to evaluate, both quantitatively and qualitatively, the influence of empathic behaviours on users' perception of a social robot. More specifically, we wanted to evaluate whether the iCat's empathic behaviours influenced the way players perceived their relationship with the robot.

\subsection{Procedure}

A between-subject experiment where participants played a chess game against each other with an iCat commenting on their games was performed. Forty subjects, 36 male and 4 female, with ages ranging from 18 to 28 years, took part in the experiment. To recruit participants for the study, an email asking for volunteers who knew how to play chess was sent to the student mailing list of the Computer Science Department at a Portuguese University. The first 40 participants who replied to this email and met the necessary requirements for the study (i.e., knew how to play chess and never interacted with the iCat before) were selected. The selected participants were assigned to a time slot (2 participants in each slot) depending on their availability. None of the subjects who played together knew each other beforehand. At the scheduled time, they were asked to sit in front of each other, having the iCat on their side commenting on the game (in Portuguese), and played an entire chess game. Figure 3 contains a picture taken during the experiment. Interactions lasted an average of one hour. At the end of the game, participants were guided to different rooms where they filled out a questionnaire and answered some open-ended questions. The initial part of the questionnaire contained some demographic questions, including participant's age and chess expertise (beginner, intermediate or advanced). 21 participants considered themselves as beginners, 17 as intermediate and 2 considered themselves to have advanced chess knowledge.

\subsection{Conditions}

The behaviour of the iCat varied whether the robot was addressing the player controlling the white or black pieces. Participants playing with the black pieces belonged to the supportive condition, where the iCat behaved towards them according to the empathic behaviours described above. Participants playing with the white pieces were part of the control condition and the robot addressed them neutrally. However, since these participants may perceive that the robot is somewhat against them, because it is treating the other player in an empathic manner, throughout the remaining of the paper this condition will be referred to as against.

\subsection{Measures}

To quantitatively measure the influence of empathic behaviours on the relationship between participants and the iCat robot, we took inspiration from the McGill Friendship Questionnaire (Mendelson and Aboud, 1999). This questionnaire quantifies the degree to which a friend fulfils the functions existing in most friendship definitions: (1) stimulating companionship - doing enjoyable or exciting things together; (2) help - providing guidance and other forms of aid; (3) intimacy - being sensitive to the other's needs and states and being open to honest expressions of 
Table 2: Questionnaire items used in this study (translated into English) with the corresponding means and standard deviations for each condition.

\begin{tabular}{|c|c|c|c|c|c|}
\hline \multirow[t]{2}{*}{ Function } & \multirow[t]{2}{*}{ Assertions } & \multicolumn{2}{|c|}{ Supportive } & \multicolumn{2}{|c|}{ Against } \\
\hline & & $M$ & $S D$ & $M$ & $S D$ \\
\hline Stimulating & I had fun playing with iCat by my side. & 4.4 & 0.5 & 4.0 & 1.2 \\
\hline \multirow[t]{4}{*}{ Companionship } & iCat behaved as my companion during the game. & 3.6 & 0.7 & 2.9 & 0.8 \\
\hline & iCat made me laugh. & 4.8 & 0.4 & 4.8 & 0.9 \\
\hline & I enjoyed talking to iCat. & 3.4 & 1.2 & 2.6 & 1.2 \\
\hline & It was nice being with iCat. & 4.0 & 0.7 & 3.8 & 0.7 \\
\hline \multirow[t]{4}{*}{ Help } & iCat helped me during the game. & 3.0 & 1.0 & 3.1 & 0.8 \\
\hline & iCat's comments were useful to me. & 3.8 & 0.8 & 3.8 & 0.7 \\
\hline & iCat showed me how to do things better. & 2.7 & 1.1 & 2.7 & 1.4 \\
\hline & iCat's comments were helpful to me. & 3.0 & 1.2 & 2.9 & 1.1 \\
\hline \multirow[t]{2}{*}{ Intimacy } & iCat knew when something was bothering me. & 2.9 & 1.2 & 2.1 & 1.0 \\
\hline & iCat knew when I was upset. & 2.9 & 1.2 & 2.1 & 0.8 \\
\hline Reliable & iCat was loyal to me. & 4.1 & 0.7 & 3.1 & 1.2 \\
\hline \multirow[t]{2}{*}{ Alliance } & iCat would still be my friend if we did not see each other for several months. & 3.6 & 1.0 & 2.7 & 1.2 \\
\hline & iCat would still be my friend even when we did not agree on something. & 4.0 & 0.8 & 3.3 & 1.1 \\
\hline Self- & iCat encouraged me to play better during the game. & 4.0 & 0.7 & 2.9 & 1.3 \\
\hline \multirow[t]{5}{*}{ Validation } & iCat praised me when I played well. & 4.8 & 0.4 & 4.3 & 0.7 \\
\hline & iCat made me feel intelligent. & 3.4 & 1.0 & 2.8 & 0.9 \\
\hline & I felt that I could play better in the presence of iCat. & 3.9 & 0.9 & 3.8 & 0.9 \\
\hline & iCat enhanced the aspects that I am good at. & 3.4 & 1.1 & 3.1 & 0.9 \\
\hline & iCat made me feel special. & 2.6 & 0.9 & 2.7 & 1.0 \\
\hline Emotional & If I were worried, iCat would make me feel better. & 3.0 & 1.0 & 2.7 & 1.2 \\
\hline \multirow[t]{2}{*}{ Security } & If I were nervous, iCat would make me feel more calm. & 3.4 & 1.1 & 3.0 & 1.4 \\
\hline & If I were upset, iCat would make me feel better. & 3.3 & 1.0 & 3.0 & 1.3 \\
\hline
\end{tabular}

thoughts, feelings and personal information; (4) reliable alliance - remaining available and loyal; (5) self-validation reassuring, encouraging, and otherwise helping the other maintain a positive self-image; and (6) emotional security providing comfort and confidence in novel or threatening situations.

A friendship relation, having similar patterns as human-human friendship, cannot be established between a human and robot, especially in an interaction lasting approximately one hour. However, as empathy is a characteristic that people value in friendships (Adams et al., 2000), we believe that by analysing the friendship functions individually, some indicators on improving long-term interaction between humans and robots can be retrieved. Thus, we hypothesise that subjects towards whom the robot displays empathic behaviour will rate some of these friendship functions more positively.

As the participants' mother language was Portuguese, we used a validated Portuguese version of the McGill Friendship Questionnaire (Souza, 2006). The questionnaire contains a set of five-point Likert scale items for each friendship function, ranging from 1 (totally disagree) to 5 (completely agree). We removed some assertions that were not applicable in this experimental set-up and slightly adapted some existing assertions to become more in tune with the type of interaction that users had with the iCat. Table 2 contains the English translation of the assertions used in the questionnaire and the corresponding average rankings that users provided for each item separated by the control conditions of the study.

In addition to the perceived friendship measure, we also investigated the overall goals and expectations of users after interacting with the iCat. To do so, we analysed participants' responses to the following open-ended questions: "I liked that iCat...", "When I played bad, iCat...", "When I played well, iCat...", "When I was feeling insecure about the game, iCat...", "What would make me interact again with iCat would be...". 


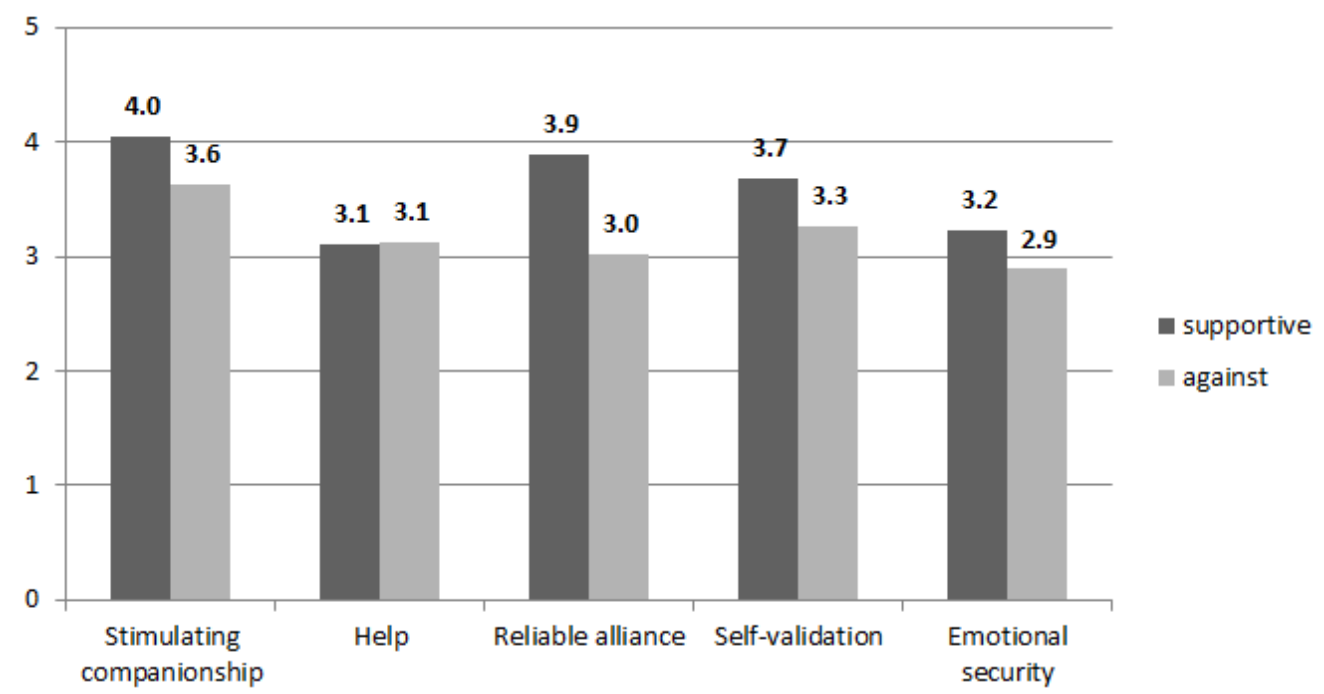

Figure 4: Mean values of the friendship functions (except intimacy) in the supportive and against conditions.

\subsection{Quantitative Data Results}

To test our hypothesis, we first performed a Cronbach's alpha test to evaluate the internal consistency of the adapted assertions of the Friendship Functions. The index was reliable for stimulating companionship $(\alpha=.79)$, help $(\alpha=.86)$, reliable alliance ( $\alpha=.69)$, self-validation $(\alpha=.8)$ and emotional security $(\alpha=.71)$, but not for intimacy $(\alpha=.56)$.

We noticed that a small group of participants took much less time to fill in the questionnaire than the majority of participants. These subjects also provided some inadequate responses to the open-ended questions and, in some cases, did not answer some questions, which suggests that they did not devote enough time to provide reliable answers. Therefore, we decided to remove these participants from the analysis. 4 participants were excluded from the supportive condition and 5 from the against condition; our final sample was 16 in the supportive group and 15 in the against group. None of the removed participants was female.

After this step, we ran a Mann-Whitney test for each friendship function ${ }^{2}$. Each function was calculated as the average of all questionnaire items associated with that friendship function.

\subsubsection{Stimulating companionship - doing enjoyable and exciting things together}

Subjects in the supportive condition rated this function significantly higher than subjects in the against condition $(U=72.5, p<0.05, z=-1.893)$. The chart in Figure 4 shows the stimulating companionship function means in each condition.

\subsubsection{Help - guidance and other forms of aid}

Figure 4 illustrates that supportive and against conditions are not significantly different with regard to the help function $(U=118, p=0.47, z=-0.79)$, suggesting that adding empathic behaviours does not seem to affect the quality of feedback provided by the robot. It is also important to note that the ratings for the questionnaire items related to help were rather low in both conditions, as depicted in Table 2.

\subsubsection{Intimacy - sensitivity to other's needs and states}

As we could not guarantee the reliability of this function, we present the results of the proposed assertions for Intimacy separately (Figure 5). The ratings of the two statements, "iCat knew when something was bothering me" and "iCat knew when I was upset", differed significantly with better ratings for the supportive condition $(U=69.5, p$ $<0.05, z=-2.058$ and $U=71.5, p<0.05, z=-2.033$, respectively).

\footnotetext{
${ }^{2}$ Non-parametric tests were used because the data did not follow a normal distribution.
} 


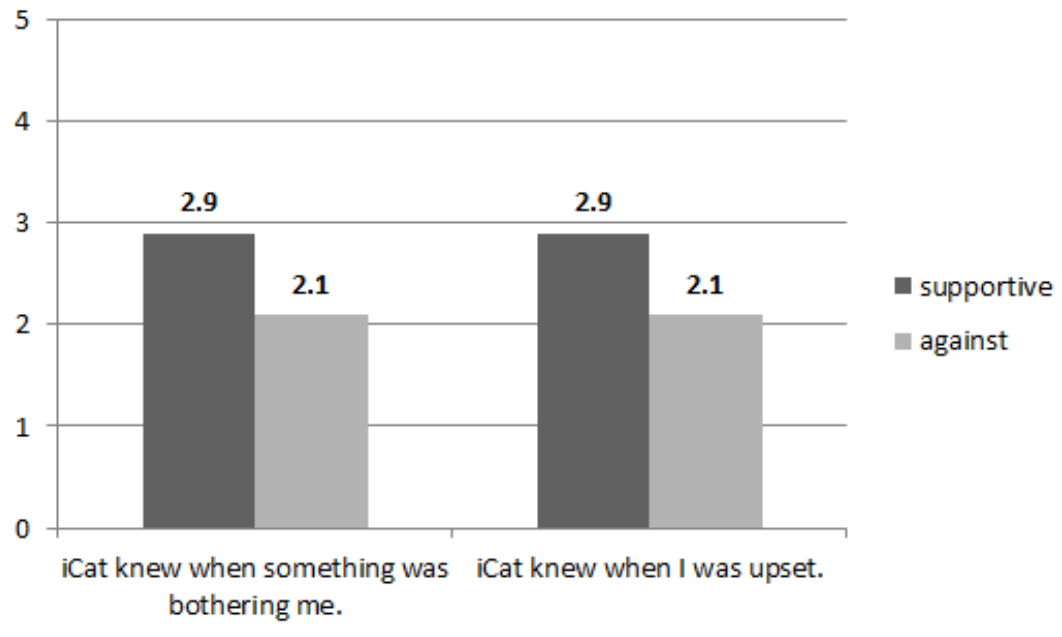

Figure 5: Mean values of the intimacy assertions in the supportive and against conditions.

\subsubsection{Reliable Alliance - remaining available and loyal}

The ratings of the reliable alliance function were significantly higher in the supportive condition than the against condition $(U=46, p<0.01, z=-2.954)$. This can also be verified in the chart displayed in Figure 4.

\subsubsection{Self-validation - reassuring, encouraging, and helping the other to maintain a positive image}

In this function, there was also a significant difference in the direction of the supportive condition $(U=66.5, p$ $<0.05, z=-2.131$ ), as illustrated in Figure 4. The questionnaire item that contributed the most for this difference was "iCat encouraged me to play better during the game" (see Table 2).

\subsubsection{Emotional security - providing comfort and confidence}

There was no significant difference between the two groups in the emotional security function $(U=95, p=0.159$, $z=-0.998)$, despite slightly lower ratings by participants in the against condition in all questionnaire items of this function. The chart in Figure 4 also reflects these results.

\subsection{Qualitative Data Results}

This subsection presents the most relevant findings collected throughout the content analysis of the open-ended questions. For each condition, participants' answers were grouped into several categories and the frequencies of these categories were analysed.

We tried to apply statistical analysis to the qualitative data, but most of the results were inconclusive mainly due to the low frequencies of the answer categories. In particular, we ran Chi-square tests, and most of the results were either non-significant or the expected frequencies were lower than 5, which violates one of the assumptions for the Chi-Square test. When the latter happened, we ran Fisher's exact test, but even so, the only statistically significant difference between groups was in the question "When I played a good move, the iCat...", in which there was a significant association between users who stated that the iCat "encouraged them" $(\mathrm{p}<0.05)$ when compared to subjects in the control group. As the main goal of the qualitative analysis was to explore and complement the results obtained in the questionnaire (validated through statistical tests), we believe that even though we could not obtain statistically significant results - mainly due to the diversity of answers, as the questions were open-ended these results are still valuable to better understand how participants perceived the robot. As we ended up with an unbalanced number of subjects, the results are also presented as percentages.

\subsubsection{I liked that iCat...}

In both groups, participants highlighted the fact that the iCat provided feedback on their moves: $50 \%$ in the supportive condition and $67 \%$ in the against condition. Some participants also enjoyed that the robot used their 
names when speaking - 3 in the supportive condition (19\%) and 2 in the against condition (13\%). In the supportive group, 8 subjects (50\%) mentioned that they liked the iCat because it encouraged them in the difficult moments of the game: "iCat knew exactly the best moves I should play, and even when the game was almost lost, it kept giving me hope to continue (...)". One participant in this condition even mentioned that the iCat elicited an empathic feeling on him: "I liked that the iCat used my name and commented on my moves. Its facial expressions and movement made me feel empathy."

\subsubsection{When I played bad, iCat...}

Most users in both conditions recognised that the robot warned them about their bad moves - $10(63 \%)$ in the supportive condition and $13(87 \%)$ in the against group. One participant in the supportive condition even answered that the iCat got sad when he played poorly. The opposite answer was given by 2 participants (13\%) in the against condition (they noticed that the iCat got happy when they played bad). 4 participants $(25 \%)$ in the supportive condition also mentioned that when they played bad moves, the robot encouraged them to play better.

\subsubsection{When I played well, iCat...}

A substantial number of subjects answered that the iCat congratulated them when they played good moves (44\% from the supportive condition and $67 \%$ from the control condition). 3 participants (20\%) from the supportive condition stated that the robot got happy when they played good moves, while the same number of subjects in the control condition said that the robot got sad. As in the previous open-ended question, 8 subjects (50\%) from the supportive condition also added that the robot gave them positive feedback: "When I played a good move, iCat demonstrated his support."

\subsubsection{When I was feeling insecure about the game, iCat...}

Nearly one-third of the participants indicated that they did not feel insecure in any part of the game and therefore did not answer this question. For those who answered, the opinions differed among conditions. While nearly half of the participants in the against group (47\%) did not notice any differences in the iCat's behaviour, 6 subjects (38\%) in the supportive group stated that the robot encouraged them when they felt insecure during the game: "When I felt insecure during the game, the iCat tried to make me calm, so I could better play my next moves." In fact, participants from the against condition recognised that the iCat supported their opponent more: "It didn't help much... I got the feeling that iCat was supporting my opponent the whole time and didn't care about me."

\subsubsection{What would make me interact with iCat again would be...}

Participants from both conditions proposed several motives for interacting again with the iCat. While in the against condition 7 subjects (47\%) would like to interact again with the robot just for fun, 6 participants (38\%) in the supportive group would like to play another game in this same setting to improve their chess skills. 7 participants stated that they would like to play against the iCat, instead of having the robot commenting on their game - 3 participants (19\%) from the supportive group and 4 (27\%) from the against condition. Additionally, 5 participants from the supportive condition (31\%) would like to play another match in this same setting, in contrast to only 2 (13\%) from the control version.

\section{Discussion and Conclusions}

This paper addressed the role of empathic behaviours on users' interactions with a social robot. Empathic behaviours reported in the literature were modelled in a social robot capable of inferring some of the user's affective states, reacting emotionally to such states and commenting on a chess game. An experiment where users interacted with such a robot was reported.

Several friendship functions were measured using a questionnaire. The results suggest that our initial hypothesis is valid for most measured friendship functions. Participants who interacted with the supportive version of the robot provided significantly higher ratings of companionship, reliable alliance and self-validation. Considering that companionship is about spending time and doing things together, these results suggest that empathic robots can provide 
more enjoyable interactions. As such, users may eventually spend more time interacting with the robot, which is important if we aim to build companions capable of engaging users in long-term interactions (Leite et al. (2010) present a deeper evaluation of this dimension). However, these assumptions must be verified by a long-term study. The results regarding reliable alliance can be explained by the iCat being empathic to the user in the supportive condition, remaining loyal to that player whether she/he is winning or losing the game. For self-validation, the encouraging behaviours that the iCat displays towards participants in the supportive version seem to have a positive effect on users from this group. Nevertheless, it is important to stress that, in both conditions, the iCat says whether the player did a good or bad move, which is also a form of reassurance. Our initial hypothesis was not validated only in help and emotional security. However, given the experimental scenario design and the interaction length, such results were not totally unexpected. The lower and non-significant different scores on help are understandable, because even though the iCat gives feedback on both players' moves (in a more empathic manner for subjects in the supportive version), it does not provide any concrete suggestions on how to play the game better. For emotional security, this may be a function that requires long-term interaction before users consider that the iCat or another entity can comfort them. Therefore, a long-term study is needed to clarify this result.

Although the results for intimacy cannot be analysed within the scope of the friendship questionnaire, the supportive group provided significantly higher ratings in the two assertions individually. As we are inferring the user's affective state considering his/her situation in the game, these results support our previous work (Castellano et al., 2009) in which we found that task-related features are important for discriminating among user's affective states.

The overall impression and understanding of the interaction was investigated by a content analysis of participants' answers to open-ended questions. This analysis revealed that subjects towards whom the iCat behaved empathically considered the robot more encouraging and sensible to their feelings. These results reinforce the findings obtained in the questionnaire, particularly that the empathic behaviours implemented in the robot were well understood and accepted by users. Some subjects in the against condition recognised that the iCat's behaviour was more supportive towards their opponent, but they still valued the robot's feedback on their moves. Players from the supportive group not only valued the robot's feedback, but they considered the robot's messages encouraging. More subjects from the supportive condition would like to interact again with the robot in this setting, and they would do so to improve their chess skills. These results indicate that empathy might be a relevant characteristic in social robots for educational purposes (e.g., robots who act as tutors).

Although this study was conducted within the context of a chess game, we believe that the obtained results can be transferable to other application domains in HRI where empathy is relevant, for example robot-assisted therapy (Tapus and Mataric, 2008) or educational robots. A result that can be generalised is that users are good at discriminating empathic behaviours conveyed through verbal and facial expressions by a social robot, which positively affects their perception of such a robot. The generic empathic model presented in Section 4 could also be adapted to other domains. For instance, consider the case of a robot that assists a patient during the rehabilitation process after a stroke. The robot could infer the possible affective state of the user by analysing the evolution of his/her recovery (e.g., by considering the percentage of recovery within each session over time). The robot could change its facial expressions and verbal utterances to motivate the user when the recovery slows down, for example, by reminding the user about the importance of completing the rehabilitation plan.

\section{Future Work}

The presented study has some limitations that must be acknowledged and addressed in future work. First, gender bias occurred in our participant pool primarily due to the unbalanced percentage of men and women playing chess: women represent less than $5 \%$ of the registered tournament players worldwide, which is less than the percentage of women in our sample (Maass et al., 2008). Additionally, subjects were graduate and undergraduate students recruited at a computer science university, where most students are male. Another limitation that deserves attention is that the experimental setup where two players (one from each condition) interact with the robot at the same time could lead to perceived signs of favouritism in the player from the supportive condition (Fogg and Nass, 1997) and, due to the situational context, ostracism in the player from the against condition (Williams, 2007). We tried to minimize this effect as much as possible, by having the robot also commenting the quality of the moves of the players in the against condition, the same number of interactions and feedback on the game for both players, and changing only the context of the messages and the iCat's facial expressions (more empathic towards the companion player). Nevertheless, 
there may be an implicit effect on the results, and we cannot guarantee that in a situation where, for example, both players received an empathic treatment or a neutral treatment, the results would be exactly the same. Therefore, all the conclusions of the study can be generalised only to a particular context, in which a subject is being subject to a certain treatment while watching another player being treated differently. Further studies are needed to verify if the results also apply to other contexts. Additionally, as several empathic behaviours were evaluated simultaneously, it is difficult to differentiate which of these empathic behaviours (e.g., facial expressions, using the person's name more often, encouraging comments, ...) contributed the most to the presented results. In general, the results indicate that empathic behaviours, including facial expressions and verbal utterances, increase people's perception of friendship towards a robot, but the behaviours that play a larger role in this effect must be determined in a future study.

For improving the robot's behaviour, a relevant aspect is the speech intonation of the robot, as the current text-tospeech lacks affective intonation. Consequently, some of the robot's utterances might not seem as empathic as they were intended to be in the scenario design. Two possible solutions to overcome this problem are to use either emotive text-to-speech or pre-recorded utterances. Also, we are currently integrating an affect recognition system into this scenario that considers not only the context of the task but also visual information from the users (Castellano et al., 2010). The robot will thus have more accurate information about the user's affective state and could react in a more appropriate and empathic manner.

\section{Acknowledgements}

This research was supported by EU $7^{\text {th }}$ Framework Program (FP7/2007-2013) under grant agreement no. 215554 and by national funds through FCT - Fundação para a Ciência e a Tecnologia, under project PEst-OE/EEI/LA0021/2011, the PIDDAC Program funds and a PhD scholarship (SFRHBD/ 41358/2007).

\section{References}

Adams, R., Blieszner, R., De Vries, B., 2000. Definitions of friendship in the third age: Age, gender, and study location effects. Journal of Aging Studies 14, 117-133.

Anderson, C., Keltner, D., 2002. The role of empathy in the formation and maintenance of social bonds. Behavioural and Brain Sciences 25 (1), 21-22.

Batson, C., Shaw, L., 1991. Evidence for altruism: Toward a pluralism of prosocial motives. Psychological Inquiry 2 (2), 107-122.

Bickmore, T., Picard, R., 2005. Establishing and maintaining long-term human-computer relationships. ACM Transactions on Computer-Human Interaction (TOCHI) 12 (2), 327.

Brave, S., Nass, C., Hutchinson, K., 2005. Computers that care: investigating the effects of orientation of emotion exhibited by an embodied computer agent. Int. J. Hum.-Comput. Stud. 62 (2), 161-178.

Breazeal, C., 2009. Role of expressive behaviour for robots that learn from people. Philosophical Transactions of the Royal Society B: Biological Sciences 364 (1535), 3527.

Breazeal, C., Berlin, M., Brooks, A., Gray, J., Thomaz, A. L., 2006. Using perspective taking to learn from ambiguous demonstrations. Robotics and Autonomous Systems 54 (5), $385-393$.

Castellano, G., Leite, I., Pereira, A., Martinho, C., Paiva, A., McOwan, P., sept. 2009. It's all in the game: Towards an affect sensitive and context aware game companion. In: Affective Computing and Intelligent Interaction, 2009. ACII 2009. 3rd International Conference on. pp. 1 -8.

Castellano, G., Leite, I., Pereira, A., Martinho, C., Paiva, A., McOwan, P. W., 2010. Affect recognition for interactive companions: challenges and design in real world scenarios. Journal on Multimodal User Interfaces 3 (1-2), 89-98.

Cooper, B., Brna, P., Martins, A., 1999. Effective affective in intelligent systems - building on evidence of empathy in teaching and learning. In: Paiva, A. (Ed.), IWAI. Vol. 1814 of Lecture Notes in Computer Science. Springer, pp. 21-34.

Cramer, H., Goddijn, J., Wielinga, B., Evers, V., 2010. Effects of (in)accurate empathy and situational valence on attitudes towards robots. In: HRI '10: Proceeding of the 5th ACM/IEEE international conference on Human-robot interaction. ACM, New York, NY, USA, pp. 141-142.

Davis, M. H., 1996. Empathy: A social psychological approach. Westview Press.

De Vignemont, F., Singer, T., 2006. The empathic brain: how, when and why? Trends in Cognitive Sciences 10 (10), 435-441.

Einsenberg, N., 2000. Empathy and sympathy. In: Lewis, M., M., H.-J. J. (Eds.), Handbook of Emotions. New York: Guildford Press, pp. 677-691.

Eisenberg, N., Fabes, R. A., 1990. Empathy: Conceptualization, measurement, and relation to prosocial behavior. Motivation and Emotion 14, 131-149.

Feshbach, N. D., 1978. Studies of empathic behavior in children. Progress in experimental personality research 8, 1-47.

Fogg, B., Nass, C., 1997. Silicon sycophants: The effects of computers that flatter. International Journal of Human-Computer Studies.

Hegel, F., Spexard, T., Vogt, T., Horstmann, G., Wrede, B., 2006. Playing a different imitation game: Interaction with an Empathic Android Robot. In: Proc. 2006 IEEE-RAS International Conference on Humanoid Robots (Humanoids06). pp. 56-61.

Hoffman, M., 1982. Development of prosocial motivation: Empathy and guilt. The development of prosocial behavior $281,313$.

Hoffman, M., 2001. Empathy and moral development: Implications for caring and justice. Cambridge Univ Press.

Ishiguro, H., 2008. Studies on humanlike robots — humanoid, android and geminoid. In: SIMPAR '08: Proceedings of the 1st International Conference on Simulation, Modeling, and Programming for Autonomous Robots. Springer-Verlag, Berlin, Heidelberg, pp. $2-2$. 
Krebs, D., 1970. Altruism: An examination of the concept and a review of the literature. Psychological Bulletin 73 (4), $258-302$.

Leite, I., Martinho, C., Pereira, A., Paiva, A., 2008. icat: an affective game buddy based on anticipatory mechanisms. In: Proceedings of the 7th international joint conference on Autonomous agents and multiagent systems-Volume 3. International Foundation for Autonomous Agents and Multiagent Systems, pp. 1229-1232.

Leite, I., Martinho, C., Pereira, A., Paiva, A., 2009. As Time goes by: Long-term evaluation of social presence in robotic companions. In: Robot and Human Interactive Communication, 2009. RO-MAN 2009. The 18th IEEE International Symposium on. IEEE, pp. 669-674.

Leite, I., Mascarenhas, S., Pereira, A., Martinho, C., Prada, R., Paiva, A., 2010. "why cant we be friends?" an empathic game companion for long-term interaction. In: Allbeck, J., Badler, N., Bickmore, T., Pelachaud, C., Safonova, A. (Eds.), Intelligent Virtual Agents. Vol. 6356 of Lecture Notes in Computer Science. Springer Berlin / Heidelberg, pp. 315-321.

Maass, A., D'Ettole, C., Cadinu, M., 2008. Checkmate? The role of gender stereotypes in the ultimate intellectual sport. European Journal of Social Psychology 38 (2), 231-245.

Martinho, C., Paiva, A., 2006. Using anticipation to create believable behaviour. In: Proceedings of the 21 st national conference on Artificial intelligence - Volume 1. AAAI Press, pp. 175-180.

McQuiggan, S. W., Lester, J. C., April 2007. Modeling and evaluating empathy in embodied companion agents. Int. J. Hum.-Comput. Stud. 65, 348-360.

Mendelson, M. J., Aboud, F. E., Apr 1999. Measuring friendship quality in late adolescents and young adults: Mcgill friendship questionnaires. Canadian Journal of Behavioural Science 31 (1), 130-132.

Paiva, A., Dias, J., Sobral, D., Aylett, R., Sobreperez, P., Woods, S., Zoll, C., Hall, L., 2004. Caring for agents and agents that care: Building empathic relations with synthetic agents. In: Proceedings of the Third International Joint Conference on Autonomous Agents and Multiagent Systems-Volume 1. IEEE Computer Society, pp. 194-201.

Prendinger, H., Ishizuka, M., 2005. The Empathic Companion: a Character-Based Interface That Addresses Users' Affective States. Applied Artificial Intelligence 19 (3-4), 267-285.

Riek, L. D., Paul, P. C., Robinson, P., 2010. When my robot smiles at me: Enabling human-robot rapport via real-time head gesture mimicry. Journal on Multimodal User Interfaces 3 (1-2), 99-108.

Rodrigues, S., Mascarenhas, S., Dias, J., Paiva, A., 2009. "I can feel it too!": Emergent empathic reactions between synthetic characters. In: Proceedings of the International Conference on Affective Computing \& Intelligent Interaction (ACII).

Saerbeck, M., Schut, T., Bartneck, C., Janse, M., 2010. Expressive robots in education: varying the degree of social supportive behavior of a robotic tutor. In: Proceedings of the 28th international conference on Human factors in computing systems. ACM, pp. 1613-1622.

Souza, L. K., 2006. Amizade em adultos: adaptaço e validao dos questionrios mcgill e um estudo de diferenas de género. Ph.D. thesis, Universidade Federal do Rio Grande do Sul.

Stephan, W., Finlay, K., 1999. The Role of Empathy in Improving Intergroup Relations. Journal of Social Issues 55 (4), $729-743$.

Stotland, E., 1969. Exploratory investigations of empathy. In: Berkowitz, L. (Ed.), Advances in experimental social psychology, Volume 4. Vol. 4. Academic Press, pp. 271-314.

Strayer, J., 1987. Affective and cognitive perspectives on empathy. In: Eisenberg, N., Strayer, J. (Eds.), Empathy and its Development. Cambridge University Press, pp. 218-244.

Tapus, A., Mataric, M., 2007. Emulating empathy in socially assistive robotics. In: Proceedings of the American Association of Artificial Intelligence (AAAI) Spring Symposium on-Multidisciplinary Collaboration for Socially Assistive Robotics.

Tapus, A., Mataric, M., 2008. Socially assistive robots: The link between personality, empathy, physiological signals, and task performance. In: AAAI Spring Symposium, Palo Alto, Stanford, USA. Vol. 8.

Torrey, C., Fussell, S., Kiesler, S., 2010. What robots could teach us about perspective-taking. In: Morsella, E. (Ed.), Expressing oneself/Expressing ones self: A festschrift in honor of Robert M. Krauss. Taylor and Francis, NY, pp. 93-106.

van Breemen, A., Yan, X., Meerbeek, B., 2005. icat: an animated user-interface robot with personality. In: AAMAS '05: Proceedings of the fourth international joint conference on Autonomous agents and multiagent systems. ACM, New York, NY, USA, pp. 143-144.

Williams, K. D., 2007. Ostracism. Annual Review of Psychology 58 (1), 425-452.

Zeng, Z., Pantic, M., Roisman, G. I., Huang, T. S., 2009. A survey of affect recognition methods: Audio, visual and spontaneous expressions. IEEE Transactions on Pattern Analysis and Machine Intelligence 31 (1), 39-58. 